MATHEMATICS OF GRAVITATION

PART II, GRAVITATIONAL WAVE DETECTION

BANACH CENTER PUBLICATIONS, VOLUME 41

INSTITUTE OF MATHEMATICS

POLISH ACADEMY OF SCIENCES

WARSZAWA 1997

\title{
ON THE QUANTAL NATURE OF THE COULOMB FIELD
}

\author{
ANDRZEJ STARUSZKIEWICZ \\ Institute of Physics, Jagiellonian University \\ Reymonta 4, 30-059 Kraków, Poland
}

\begin{abstract}
It is shown that the total electric charge, as determined from the Gauss law, is a quantum object. The argument is based on elementary considerations concerning the number of photons, which should be large in a classical situation.
\end{abstract}

1. The quantum nature of the electric charge. I think I should justify first of all why I am talking at this Workshop, devoted, as it is, to the classical and quantum theory of gravity. It is really amazing that so many people work on the quantum theory of gravity while no one seems to be interested in the quantum theory of electromagnetism. It is commonly accepted nowadays that the theory known as Quantum Electrodynamics (QED) is an incredibly successful calculational scheme but not a physical theory. There are many arguments supporting this point of view but one is especially relevant for me: QED, as Feynman observed in his Nobel Lecture and repeated in his popular book [1], is unable to explain the phenomenon of charge quantization i.e. the oldest, the most elementary, and the most spectacular manifestation of the quantum nature of electricity. Nothing even approximately so spectacular is known for gravity. Hence the phenomenon of so many people working on quantum theory of gravity is certainly curious and can be only explained by Descartes' saying that "most people prefer to speculate upon difficult things rather than to arrive at the truth in very simple things" [2].

I will start my consideration of the Coulomb field with the criterion of applicability of the classical field concept given by Berestetsky, Lifshitz and Pitaevsky [3]. The electromagnetic field $F_{\mu \nu}$ is approximately classical, if $(\hbar=1=c)$

$$
\left(\Delta x^{0}\right)^{2} \sqrt{F_{01}^{2}+F_{02}^{2}+F_{03}^{2}} \gg 1,
$$

where $\Delta x^{0}$ is the observation time, over which the field can be averaged without being significantly changed. For a static field this time is obviously infinite and therefore,

1991 Mathematics Subject Classification: 81P05.

The paper is in final form and no version of it will be published elsewhere. 
conclude Berestetsky, Lifshitz and Pitaevsky, "a static field is always classical" (op. cit., page 30$)$.

This conclusion is sometimes evidently correct. Consider the hydrogen atom as described by the Dirac equation. The theory of the hydrogen atom is obtained by putting the classical proton field into the equation. The result is in perfect agreement with observations, which shows that the proton Coulomb field is indeed completely classical, it is as classical as the book you have in hand. Or even more so, since in the case of the hydrogen atom we have very precise, quantitative comparisons of theory and experiment, which do not exist in the case of the book.

However, if the conclusion of Berestetsky, Lifshitz and Pitaevsky is always correct, then the phenomenon of charge quantization presents an insoluble dilemma: where the Planck constant $\hbar$ comes from into the expression for the elementary charge?

I will show that the conclusion is not always correct. As the first example I consider so called zero frequency fields i.e. fields emitted when a charge changes its four-velocity. Suppose that the point charge $Q$ is scattered at the origin of the coordinate system, its world line being

$$
x^{\mu}(s)= \begin{cases}u^{\mu} s & \text { for } s<0, \\ w^{\mu} s & \text { for } s>0 .\end{cases}
$$

The radiation, defined after Dirac as the difference between the retarded solution and the advanced solution, vanishes both in the future light cone and in the past light cone. Outside the light cone it is equal to the difference of two Coulomb fields moving with four-velocities $u$ and $w$, respectively:

$$
A_{\mu}(x)=Q \Theta(-x x)\left\{\frac{u_{\mu}}{r(u)}-\frac{w_{\mu}}{r(w)}\right\} .
$$

Here $\Theta$ is the Heaviside step function, $x x=\left(x^{0}\right)^{2}-\left(x^{1}\right)^{2}-\left(x^{2}\right)^{2}-\left(x^{3}\right)^{2}$, and $r(u)$ is the distance from the origin at the rest frame of $u$ :

$$
r(u)=\sqrt{(u x)^{2}-(u u)(x x)} .
$$

The important point is that the field vanishes exactly inside the future and past light cone. This means that the averaging time $\Delta x^{0}$ cannot exceed, in a given reference frame, $2 r$ :

$$
\left|\Delta x^{0}\right|<2 r .
$$

The radiation field is simply the Coulomb field $Q / r^{2}$ multiplied by a kinematical, velocity and angle dependent factor, which is clearly irrelevant for the present consideration and which can be made of order 1 by a suitable choice of the velocity change. Thus the criterion of Berestetsky, Lifshitz and Pitaevsky takes on the form

$$
\begin{aligned}
& (2 r)^{2} \frac{|Q|}{r^{2}} \gg 1 \\
& \text { i.e. }|Q| \gg \frac{1}{4},
\end{aligned}
$$

which certainly does not hold for a single electron, for which

$$
|Q|=\frac{1}{\sqrt{137}}<\frac{1}{4} .
$$


We see that the electron charge, as manifesting itself in the magnitude of zero frequency fields, is a deeply nonclassical object.

My second example is directly related to the most obvious difficulty which one encounters when one tries to quantize both electromagnetic and gravitational field: the existence of constraints. The constraint equation

$$
\operatorname{div} E=4 \pi \rho
$$

means that the total charge of an isolated system can be computed as an integral over the sphere at the spatial infinity,

$$
Q=\int_{\Sigma} \rho d V=\frac{1}{4 \pi} \int_{\partial \Sigma} E \cdot d S
$$

$\Sigma$ is an arbitrary Cauchy surface i.e. a space-like surface intersected at exactly one point by each time-like and null straight line. This means, however, that if we start our Cauchy surface at the point $P$, then it must remain entirely outside the light cone of $P$. Therefore, the averaging time $\Delta x^{0}$ is not infinite but again limited by the opening of the light cone,

$$
\left|\Delta x^{0}\right|<2 r,
$$

and the criterion of Berestetsky, Lifshitz and Pitaevsky gives again

$$
|Q| \gg \frac{1}{4}
$$

which certainly does not hold for a single electron. The electron charge, as determined from the Gauss law, is a deeply nonclassical object, at least for the experimentally observed value of the fine structure constant.

This argument resolves, I believe, a contradiction between two indubitable facts, the fact that the proton charge in the hydrogen atom is a completely classical object and the fact that its magnitude contains the Planck constant $\hbar$.

2. The structure of the electromagnetic field at the spatial infinity. If the electromagnetic field $F_{\mu \nu}(x)$ has a nonvanishing total charge, then it must be homogeneous of degree -2 at the spatial infinity,

$$
F_{\mu \nu}(\lambda x)=\lambda^{-2} F_{\mu \nu}(x)
$$

for all $\lambda>0$ and for $x x \rightarrow-\infty$. Thus we can investigate field at the spatial infinity by investigating homogeneous of degree -2 solutions of the Maxwell equations.

Assume that

$$
A_{\mu}(\lambda x)=\lambda^{-1} A_{\mu}(x) \text { for each } \lambda>0 .
$$

One can easily show, using the Maxwell equations and the above homogeneity condition, that both $F_{\mu \nu}(x) x^{\nu}$ and $(1 / 2) \varepsilon^{\mu \nu \rho \sigma} x_{\nu} F_{\rho \sigma}(x)$ are gradients of homogeneous of degree zero functions,

$$
F_{\mu \nu}(x) x^{\nu}=\partial_{\mu} e(x), \frac{1}{2} \varepsilon^{\mu \nu \rho \sigma} x_{\nu} F_{\rho \sigma}(x)=\partial^{\mu} m(x) .
$$

These two functions were first discovered by Alexander and Bergmann [4]. $e(x)$ and $m(x)$ stand for the "electric" part and the "magnetic" part, respectively. $e(x)$ can be easily 
calculated:

$$
\begin{aligned}
F_{\mu \nu}(x) x^{\nu} & =\left[\partial_{\mu} A_{\nu}(x)-\partial_{\nu} A_{\mu}(x)\right] x^{\nu} \\
& =\partial_{\mu}\left[A_{\nu}(x) x^{\nu}\right]-\delta_{\mu}^{\nu} A_{\nu}(x)-x^{\nu} \partial_{\nu} A_{\mu}(x) \\
& =\partial_{\mu}\left[x^{\nu} A_{\nu}(x)\right]
\end{aligned}
$$

since

$$
x^{\nu} \partial_{\nu} A_{\mu}(x)=-A_{\mu}(x)
$$

from the Euler theorem on homogeneous functions. Hence

$$
e(x)=x^{\mu} A_{\mu}(x)
$$

up to an irrelevant constant.

The function $e(x)$ is gauge invariant. This is clear from the definition, which involves the tensor $F_{\mu \nu}(x)$ only, but may also be checked directly: in the gauge transformed potential $A_{\mu}(x)+\partial_{\mu} f(x)$ the function $f(x)$ must be homogeneous of degree zero; hence $x^{\mu} \partial_{\mu} f(x)=0$, again from the Euler theorem on homogeneous functions.

I maintain that $m(x)=0$. This is an argument against the existence of magnetic monopoles which I gave in [5] and repeat here for completeness.

Let us calculate the Lagrange function

$$
-\left[d x^{0} d x^{1} d x^{2} d x^{3}\right] F_{\mu \nu} F^{\mu \nu}
$$

for a homogeneous of degree -2 field $F_{\mu \nu}$. The result is

$$
\begin{aligned}
& -\left[d x^{0} d x^{1} d x^{2} d x^{3}\right] F_{\mu \nu} F^{\mu \nu} \\
& =2 \frac{d \sqrt{-x x}}{\sqrt{-x x}}\left[d \xi^{1} d \xi^{2} d \xi^{3}\right] \sqrt{g}\left(g^{i k} \frac{\partial e}{\partial \xi^{i}} \frac{\partial e}{\partial \xi^{k}}-g^{i k} \frac{\partial m}{\partial \xi^{i}} \frac{\partial m}{\partial \xi^{k}}\right) .
\end{aligned}
$$

Here $\xi^{1}, \xi^{2}, \xi^{3}$ are arbitrary internal coordinates on the surface $x x=-1$ while $g_{i k}$ is the metric of this surface in these coordinates.

The correctness of this equation can be seen from the following remark. Each coordinate transformation is locally a linear transformation which preserves the signature of each quadratic form. The signature of the quadratic form on the left hand side is $(+++---)$ :

$$
-F_{\mu \nu} F^{\mu \nu}=2\left(F_{01}^{2}+F_{02}^{2}+F_{03}^{2}-F_{23}^{2}-F_{31}^{2}-F_{12}^{2}\right) .
$$

Therefore, the quadratic form on the right hand side has to have the same signature. But the signature of both invariants, $g^{i k} \partial_{i} e \partial_{k} e$ and $g^{i k} \partial_{i} m \partial_{k} m$, is fixed by the signature of the internal metric $(+--)$. The only way to obtain the quadratic form with the signature $(+++---)$ from two quadratic forms with the signature $(+--)$ is to take their difference.

It is thus seen that the Lagrangian of the asymptotic field is a difference of two identical Lagrangians. Thus only one of them can have the correct sign i.e. the sign which makes the kinetic term positive. The part with the right sign is called electric, the part with the wrong sign is called magnetic and must be put equal to zero. We have here a local, i.e. seen in a point, difference between electricity and magnetism which excludes asymptotic fields of magnetic type. 
3. Quantum mechanics of the electric charge. We have seen in the first section that the total electric charge, as determined from the Gauss law, is a quantum object, at least for the actually observed value of the fine structure constant. Hence there must be something which does not commute with the total charge. In the QED this is the phase of the second quantized wave function [6]:

$$
[Q, S(x)]=i e,
$$

where $e$ is the constant which appears in the gauge invariant quantity $e A_{\mu}(x)+\partial_{\mu} S(x)$. At the spatial infinity the entire content of Maxwell's theory is reduced to the function $e(x)=x^{\mu} A_{\mu}(x)$. Hence, the phase $S(x)$ must be proportional to $e(x)$. I put forward the hypothesis [6] that

$$
S(x)=-e x^{\mu} A_{\mu}(x) .
$$

There are several arguments which support this hypothesis. Let me give just one.

Take the Coulomb field of the charge $Q$ at rest:

$$
A_{0}=\frac{Q}{r}, A_{1}=A_{2}=A_{3}=0 .
$$

Its phase, according to our hypothesis, is

$$
S(x)=-e x^{\mu} A_{\mu}(x)=-e Q \frac{t}{r} .
$$

During the eternity of time available at the spatial infinity,

$$
-r<t<r,
$$

the phase $S(x)$ changes from $e Q$ to $-e Q$. Take now the hydrogen atom with the nuclear charge $Q$ and the electron charge $e$ and assume that the radius of its circular orbit tends to infinity. During the eternity of time available,

$$
-r<t<r,
$$

the electromagnetic phase of the electron wave function,

$$
-e \int A_{\mu}(x) d x^{\mu}
$$

will change by the same amount,

$$
-e \int_{-r}^{r} \frac{Q}{r} d t=-2 e Q .
$$

Thus, the phase $S(x)=-e x^{\mu} A_{\mu}(x)$ changes as the true phase of the electron wave function in an infinitely large hydrogen atom.

The two equations

$$
\begin{gathered}
{[Q, S(x)]=i e,} \\
S(x)=-e x^{\mu} A_{\mu}(x),
\end{gathered}
$$

form together a closed theory which, I think, deserves the name of the Quantum Mechanics of the Electric Charge. It is a purely kinematic theory and thus akin to the theory of angular momentum. 


\section{References}

[1] R.P. Feynman, QED, Princeton University Press, 1985.

[2] René Descartes, Regulae Ad Directionem Ingenii, I quote from the Polish translation, published by PWN, Warsaw 1958, page 9 .

[3] W.B. Berestetsky, E.M. Lifshitz, and L.P. Pitaevsky, Relativistic Quantum Theory, Moscow 1968, in Russian.

[4] M. Alexander and P.G. Bergmann, Foundations of Physics 14, 925 (1984).

[5] A. Staruszkiewicz, an essay in honour of Yakir Aharonov, published in Quantum Coherence and Reality, ed. by J.S. Anandan and J.L. Safko, World Scientific, Singapore 1994, page 90 .

[6] A. Staruszkiewicz, Ann. Phys. (N.Y.) 190, 354 (1989). 